\title{
Portafolios electrónicos y educación superior en España: Situación y tendencias
}

\author{
Electronic portfolios and higher education in Spain: \\ Situation and trends \\ Elena Barberà \\ Universitat Oberta de Cataluña, Barcelona, España \\ ebarbera@uoc.edu \\ Adriana Gewerc Barujel \\ Universidad de Santiago de Compostela, Santiago de Compostela, España \\ adriana.gewerc@usc.es \\ José Luis Rodríguez Illera \\ Universidad de Barcelona, Barcelona, España \\ jlrodriguez@ub.edu
}

\begin{abstract}
Resumen
En el marco de la consolidación de la RED de portafolios electrónicos (e-portafolios) en el estado español, el presente artículo expone, los marcos conceptuales que guían las diferentes propuestas en el diseño y la implementación de e-portafolios en la educación superior. En este texto se realiza una cierta revisión sobre estas perspectivas teóricas. También se aborda genéricamente las tipologías de e-portafolios que se relacionan con el conocimiento práctico dirigido por un enfoque de desarrollo competencial y se finaliza el artículo apuntando líneas de desarrollo y aplicación futuras que emergen de nuevas demandas y necesidades en el campo educativo y social.
\end{abstract}

Palabras clave: E-portafolio del estudiante, evaluación basada en competencias, aprendizaje reflexivo, enseñanza y eportafolios.

\begin{abstract}
In the frame of the network of electronic portfolios (e-portfolios) in Spain, this article presents the conceptual frameworks that guide the various proposals regarding the design and implementation of e-portfolios in higher education. Then we make a revision of these theoretical perspectives and also the generic types of e-portfolios that are related to practical knowledge in the skills approach.

The article ends by recommending lines of future development and application of emerging from new demands and needs in education and social development.
\end{abstract}

Keywords: Student e-portfolio, assessment; reflexive learning, teaching and e-portfolio.

\section{PRESENTACIÓN}

Este monográfico responde a un doble objetivo. Por un lado, se pretende mostrar el estado de la cuestión de este tópico mientras se ofrece material relevante y actualizado sobre la concepción y aplicación de portafolios electrónicos (e-portafolios) en educación superior en el estado español. Por otro lado, dar visibilidad a una comunidad estable de docentes e investigadores que comparte inquietudes sobre la evolución de los e- 
portafolios en el marco educativo y que en estos momentos aglutina un porcentaje muy elevado de las prácticas que se realizan en este país ${ }^{1}$.

Ambos objetivos están obviamente conectados y, aunque los contenidos de las diferentes aportaciones concretas sobre e-portafolios configuran el núcleo de este monográfico, queremos aprovechar esta oportunidad para presentar a la comunidad de educativa, los miembros que trabajan sobre esta temática de un modo intensivo.

Si bien ya hace algún tiempo que salen a la luz iniciativas individuales en distintos puntos geográficos del estado español, no es hasta el pasado año 2006 que se inicia una Red temática conjunta que congrega la mayor parte de los grupos de investigación e innovación que trabajan de una manera significativa en esta temática en el estado.

Así bajo el auspicio del Ministerio de Educación y Ciencia, se forma la primera red nacional dedicada al desarrollo y avance de los portafolios electrónicos (http://www.redportfolio.org). La RED e-portafolio nace con vocación de aprendizaje mutuo entre investigadores aunando esfuerzos y compartiendo inquietudes a través de las experiencias iniciadas entre los participantes, a la vez que se determina crear una verdadera comunidad que ofrezca una reflexión sólida acompañada de elementos fiables enfocados a una práctica racional del e-portafolio (recursos prácticos, criterios de aplicación, referencias contrastadas, etc.) a todos los interesados que se acerquen a esta temática. La red la conforman 16 equipos de investigación de 13 universidades, el Instituto Cervantes y 7 empresas privadas distribuyendo su presencia en un total de 9 comunidades autónomas.

En su primer año, se ha dedicado a sentar las bases de la propia comunidad y al mismo tiempo a elaborar completa base de datos de las experiencias recogidas en las diferentes comunidades autónomas (se han contabilizado hasta setenta iniciativas relevantes). Y en el segundo año de vida, se consolida un observatorio permanente que va registrando el comportamiento y la vitalidad de estas y otras experiencias a través de informes regulares que se ofrecen de manera pública.

La presencia del e-portafolio en el contexto educativo es, por lo que respecta a la educación superior actual, una necesidad más que una novedad, por esa razón, con las reflexiones y experiencias prácticas que se presentan en estás páginas electrónicas se quiere contribuir al conocimiento ponderado del e-portafolio, al mismo tiempo que se anima a su uso, desarrollo racional y ajustado de esta potente herramienta verdaderamente centrada en el estudiante y basada en la publicación de evidencias de aprendizaje.

\section{INTRODUCCIÓN}

Esta parte introductoria del monográfico que presentamos tiene como objetivo mostrar una aproximación general a las definiciones sobre e-portfolios que se enmarcan en el contexto de las enseñanzas en la universidad española. De allí que en el título hagamos

\footnotetext{
${ }^{1}$ RED temática Portafolios electrónicos. Referencia: SEJ2006-27543-E/EDUC. (Ministerio de Educación y Ciencia.
}

Portafolios electrónicos y educación superior en España: Situación y tendencias. 
mención a la situación y las tendencias que hoy por hoy pueden dibujarse en el escenario educativo en el que nos movemos.

Indudablemente, debemos circunscribir el desarrollo de una herramienta didáctica, como es el e-portafolio, en el contexto global en el que se desarrolla y no es posible, por lo tanto, alejarse del significado que ha adquirido en el último tiempo a raíz de las demandas que provienen del Espacio Europeo de Educación Superior. Es ese contexto el que, de alguna manera, está popularizando una herramienta que ya tiene una larga historia de desarrollo, y que ahora se adopta y adapta para dar respuestas a necesidades complejas que provienen de los procesos de convergencia.

Es ese contexto el que reclama retos y cambios al sistema educativo en general y al universitario en particular y es allí donde una herramienta como esta, se muestra lo suficientemente flexible para ofrecer alternativas a las modalidades de enseñanza y evaluación que están vigentes y que requieren algún tipo de replanteamiento.

Ahora bien, también sabemos que una herramienta por si sola no puede generar los cambios necesarios y posibles, aunque, en el mejor de los mundos pueda ayudar y hasta estimularlos. En este caso en particular, la flexibilidad de la herramienta también da cuenta de diferentes enfoques o teorías de enseñanza y aprendizaje que pueden ayudar a configurar un tipo de e-portafolios o una particular manera de utilización. De allí que sea necesaria una conceptualización que nos ayude a comprender cada propuesta. En este artículo intentamos aproximarnos a algunas perspectivas que están vigentes tanto en el contexto del aprendizaje como de la enseñanza. Se apunta a una perspectiva cognitiva-constructiva del aprendizaje, haciendo hincapié en el valor y adecuación del conocimiento práctico, como las estructuras que se ponen en acción para la resolución de problemas. El portafolios sería un ámbito en el que se propiciaría el desarrollo de las competencias implicadas en dicho proceso.

También se muestran diferentes posibilidades o modelos de enseñanza que estarían apuntando a su vez a distintas maneras de concebir el conocimiento y el aprendizaje al mismo tiempo. De allí que se expone en el texto la posibilidad de observar qué portafolios se solicita al alumnado, y deducir en ello, qué modelo o perspectiva de enseñanza se desarrolla. El esquema que se presenta más adelante, pretende ser un punto de partida para la reflexión y el análisis de la enseñanza que desarrollamos y de la manera como utilizamos las diferentes herramientas.

Por último, al final del artículo planteamos líneas de acción futuras en el desarrollo de eportafolios. Ya que entendemos que es importante incorporar a su análisis algunas posibilidades que surgen alrededor del software que lo sustenta. Cuando hablamos de eportafolios, debemos comprender que hay una combinación-integración entre la propuesta educativa y la selección de la tecnología adecuada para llevarla adelante.

\section{E-PORTAFOLIOS EN EL CONTEXTO UNIVERSITARIO}

\subsection{Eco del e-portafolio en el ámbito universitario actual}

El e-portafolio ofrece una respuesta integrada a los requerimientos y las esperanzas que hay puestas desde la Unión Europea, cuando impulsa la creación de un nuevo marco

Portafolios electrónicos y educación superior en España: Situación y tendencias. 
educativo delineado por la convergencia hacia el Espacio Europeo de Educación Superior. Podemos decir que se trata de un sistema que se coloca al servicio de estos objetivos, puesto que el e-portafolio existía anteriormente a la iniciativa de espacio común de educación superior y, consecuentemente, no es tanto que el e-portafolio se haga eco de dichas necesidades educativas, sino que se le sitúa ahora a su servicio en este momento de cambio en el que pocas herramientas e instrumentos aparecen tan completos y versátiles para dar respuesta a la complejidad de la propuesta europea. De ahí que sea tan remarcable y notoria su presencia en la educación superior cuando es un sistema que ya se utilizaba con toda normalidad en el ámbito educativo hace más de veinte años (Wiedmer, 1998; Morris y Buckland, 2000).

Como es por todos conocido, en este marco de convergencia europea se prioriza, por un lado, el establecimiento de un sistema de enseñanza que favorezca la formación integral de los estudiantes con el fin de conseguir la óptima superación de las necesidades de la exigente realidad social y laboral que demanda nuevos perfiles competenciales y conocimientos permanentemente actualizados a lo largo del ciclo vital (Barrett y Wilkernson, 2004). Por otro lado, también se considera la reformulación de las metodologías aplicadas en las aulas centrando su énfasis en el proceso de aprendizaje y dotando de un mayor protagonismo a los estudiantes.

En este complejo contexto, a caballo entre el ámbito formativo y profesional, ya queda afianzando el cambio de orientación de una universidad que se fundamenta en un enfoque competencial reafirmando, en un primer plano, unos aprendizajes de índole personal y profesional al mismo tiempo. Bajo esta visión evolutiva marcada por el proceso de transformación educativa cobra una especial relevancia procesos de demostración y comunicación de los aprendizajes realizados a través del tiempo. Y son estos procesos socialmente argumentativos y educativamente más evolucionados los que exigen un replanteamiento de naturaleza no sólo estructural, sino de tipo más semántico que apunte al significado más profundo de las metodologías docentes. Uno de los requerimientos de cambio proviene de la fuerte implicación que asumirían los estudiantes al tener que aumentar su conocimiento sobre su propio proceso de aprendizaje, tendiendo hacia la promoción de una evaluación basada en competencias de aprendizaje.

Bajo este panorama social y educativo, para muchos, el portafolio electrónico se muestra como un sofisticado receptáculo que da respuesta a uno de los mayores retos que la educación superior tiene planteado y que resume apelando a la función acreditativa de la evaluación de los aprendizajes universitarios afín a perfiles profesionales específicos. Receptáculo puesto que acoge, contiene y demuestra los aprendizajes más relevantes de los estudiantes pero también porque resuelve técnicamente muchos de los anhelos administrativos que abarca esta ambiciosa propuesta europea como son la convalidación de aprendizajes previos, la facilitación de la movilidad entre ciudadanos europeos y el impulso de optimizar aprendizajes que no sean tan efímeros y difícilmente actualizables. Pero la implementación de un sistema de enseñanza y aprendizaje en educación superior basado en la implementación de portafolios electrónicos va más allá de esta vocación que se le presupone como intentamos determinar en los siguientes apartados.

\subsection{Tipologías de uso del e-portfolio en la universidad}

Portafolios electrónicos y educación superior en España: Situación y tendencias. Elena Barberà et al.

Página 4 de 12 
El e-portafolio explica una parte significativa de la vida del usuario, sea esta académica, profesional, personal, etc. y se remonta y alarga tanto como el usuario decida: desde la escuela primaria hasta el final de la misma vida, pasando por distintos momentos que documentan la historia de esa persona. Hemos observado que existe todavía una cierta confusión en cuanto a los propósitos de los e-portafolios que de hecho, es una de las primeras cuestiones que se deben determinar. En muchos casos concurren en un mismo tipo, por ejemplo, e-portafolios regidos por el testimonio de habilidades profesionales que se consideran afianzadas, o por la exposición de los hitos conseguidos en forma de currículo personal, o por la demostración de los mejores trabajos académicos, o por la declaración argumentada de desarrollo y crecimiento personal, entre muchos otros propósitos. Todo ello es válido, pero precisa de una ordenación hacia un objetivo más definido y, en el supuesto que se quisiera integrar todo este material diverso sería necesario una coordinación más transversal que potencie más que confunda las metas propuestas.

Específicamente, el e-portafolio universitario es una herramienta que contiene los logros más relevantes de un estudiante en este periodo vital; como periodo formativo decisivo y relacionado con la profesión se prevé que este periodo ocupe una parte importante de un portafolio más extenso. A su vez no todos los portafolios electrónicos tienen un uso similar en el ámbito de la educación superior. Si bien se idearon para mostrar los logros finales y más tarde se adaptaron para acompañar el proceso de enseñanza y aprendizaje entendido como un camino progresivo de mejora cognitiva, es el uso evaluativo el que prima en nuestros días en las universidades europeas. La dificultad de captar algo tan complejo para las instituciones universitarias como las competencias, el inapelable proceso de acreditación para la homologación de estudios y la movilidad de personas hacen del e-portafolio un instrumento de uso mayoritariamente evaluativo en estos contextos universitarios.

\section{E-PORTAFOLIOS en la enseñanza universitaria}

Si bien, como ya mencionamos anteriormente, el uso masivo de los eportafolios en el ámbito universitario se está dando en el contexto de evaluaciones complejas, también es cierto que en este contexto también se utilizan con diferentes enfoques y perspectivas. $\mathrm{Y}$ esto es debido, seguramente, a la flexibilidad de una herramienta que puede ser utilizada en diferentes contextos y también con propósitos dispares. Un portafolio (ya sea en su versión digital o analógica) puede ser una simple colección de los mejores trabajos del estudiante; un conjunto de evidencias que permita demostrar el progreso a través del tiempo; una estrategia de aprendizaje; un repositorio de materiales recolectados para la evaluación, un espacio para el desarrollo profesional... Hay diferentes maneras de definirlo y quizás esa es una explicación a las dificultades que se vislumbran en la comprensión de su significado. Para todas ellas hay objetivos específicos en mente que dependen de las concepciones de enseñanza y aprendizaje que la sustenten.

De modo general, podemos decir, que se trata de una colección de documentos que pueden ser mostrados como evidencias del proceso de aprendizaje y los logros de un sujeto. En ese contexto tiene la doble función de:

Portafolios electrónicos y educación superior en España: Situación y tendencias. 
- recoger y reflejar las experiencias de aprendizaje y logros más significativas de una persona (estudiante, profesional, trabajador...) de forma continuada.

- Informar de forma clara sobre el nivel de competencia y de otras experiencias importantes a lo largo de su aprendizaje o de su carrera.

En suma, según Barrett (1998), el portafolio es una amplia colección del trabajo del estudiante que muestra su esfuerzo, progreso y logros. Ahora bien, circunscribir una herramienta como e-portfolios en el contexto del proceso de enseñanza y de aprendizaje, necesariamente nos obliga a comenzar a pensar en las teorías que están sustentando las diferentes propuestas. Como veremos más adelante en el conjunto de este monográfico, no hay una sola manera de encarar el trabajo alrededor del eportafolios y no cabe la menor duda que esto se debe a las perspectivas teóricas que sustentan las propuestas de enseñanza, aprendizaje y evaluación que se llevan adelante. Cualquier estrategia, está apoyada en la adaptación o incorporación de teorías que le dan sustento.

Recordemos que Shulman ha planteado al portafolios como un acto teórico, y con esto esta dando pie a la afirmación de que "cada vez que se diseña, organiza o crea en el programa de formación docente una plantilla, un esquema o un modelo para un portafolio didáctico, se realiza un acto teórico" (Shulman, 1999:45). Por lo tanto, serán las teorías que se sostengan acerca de la enseñanza y el aprendizaje la que determinen la forma y el contenido que adopte el portafolio.

Se trata, como diría Litwin (1997) de analizar cuáles son las configuraciones didácticas con las que se desarrolla la enseñanza,

"que se expresan en el tratamiento de los contenidos, su particular recorte, los supuestos que maneja respecto del aprendizaje, la utilización de prácticas metacognitivas, los vínculos que establece en la clase con las prácticas profesionales involucradas en el campo de la disciplina de que se trata, el estilo de negociación de significados que genera, las relaciones entre la práctica y la teoría que incluyen lo metódico y la particular relación entre saber y el ignorar". (Litwin, 1997:97).

Las decisiones que se tomen en relación a cada uno de estos elementos, están mostrando las perspectivas teóricas que como docente estamos sustentando. Perspectivas que responden a cómo percibimos que los sujetos aprenden y también a la concepción sobre el conocimiento disciplinar y a su construcción. La enseñanza se transforma así en un referente complejo en donde se ponen en juego experiencias pasadas, teorías sustentadas, conocimiento práctico, una organización del espacio y del tiempo institucional,... en suma, un conjunto de elementos que nos ayudan a establecer ese puente entre el conocimiento que valoramos y el alumnado.

En ese contexto, el e-portafolios no puede entrar de manera aislada; como en cualquier situación estructurada, la intromisión de un nuevo elemento mueve el resto, lo reconfigura y requiere cambios. En este caso, se trata de una herramienta que, sobre todo si es utilizada como instrumento de evaluación, ayuda a repensar la totalidad o parte de la propuesta y a resignificarla en función de la nueva situación. Además, una herramienta de la potencia de ésta, nos marca un camino para su apropiación. Con esto estamos queriendo decir que, si bien es maleable y se adapta a las características que la 
propia propuesta determina en función de las opciones metodológicas, o las características que impone la disciplina que se está enseñando, la propuesta de portafolios encierra en si misma un discurso que no podemos obviar y obliga a pensar su coherencia con la propuesta de enseñanza que se viene realizando.

La mayoría de los investigadores en el campo coinciden a grandes rasgos en que el sistema de e-portafolio en contextos de enseñanza se caracteriza por:

1. el control del mismo pasa al sujeto de aprendizaje, de allí que se predisponga a una personalización del proceso. Y,

2. la posibilidad de compartir las experiencias de aprendizaje con otros y en diferentes contextos (académico, laboral, o comunitario)

Por esta razón, la misma idea de portafolios, estaría marcando un enfoque de enseñanza que pretende que los sujetos desarrollen aprendizajes profundos, estableciendo relaciones entre las experiencias de aprendizaje que se desarrollan en varios contextos y no sólo en el salón de clase. Ya que el énfasis está puesto en la integración de experiencias de aprendizaje sin importar donde estas ocurran.

Esta perspectiva incluye tres elementos: reflexión, comunicación y colaboración que reflejan que la herramienta tiene como marco de sugerencia una perspectiva cognitiva-constructiva del aprendizaje que se desarrolla sobre bases sociales, contextuales y con experiencias que expresan la actividad del sujeto. Esto supone desafíos para profesores y alumnos, ya que demanda el replanteamiento de roles hacia una perspectiva de colaboración en el proceso de aprendizaje con una responsabilidad también compartida. La reflexión ocupa aquí un lugar sustancial, porque provee al sujeto de herramientas para autoevaluarse y de allí que sugiera habilidades metacognitivas que le ayudan a aprender con autonomía a través de la vida.

Es en este contexto, donde se atribuye una especial significación al conocimiento práctico, sobre todo en un ámbito altamente formalizado como es el universitario.

\subsection{El conocimiento práctico en la universidad}

Apuntamos a un conocimiento práctico (y no tanto aplicado) como el conjunto de los conocimientos que hacen alusión a estructuras complejas de procesos que las personas ponen en acción-creación para resolver problemas o realizar actividades diversas de la vida cotidiana y del ámbito profesional orientadas a la transformación y construcción de la realidad (Barberà, 2008). En las coordenadas gobernadas por la evaluación de los aprendizajes, que es el campo natural en el que ha aterrizado el sistema e-portafolios, cabe organizar las dimensiones que la constituyen. De otro modo se desatenderían las potencialidades con las que irrumpe y se perdería esta posibilidad histórica en la que nos encontramos que ofrece la oportunidad de que el estudiante sea el centro de su propio aprendizaje.

Así, sucintamente, además de las dimensiones referidas a la acreditación y la parte más formativa ya apuntadas, se añadirían las de autorregulación y de convalidación de conocimientos previos (Blackburn y Hakel, 2006). Dos nuevas dimensiones de naturaleza muy distinta que en el ámbito universitario transnacional se

Portafolios electrónicos y educación superior en España: Situación y tendencias. 
auguran especialmente relevantes para el desarrollo de una docencia de calidad. Mientras que la primera hace referencia a un aprendizaje centrado no únicamente en la acumulación de conocimientos declarativos, sino el desarrollo de las posibilidades que posee cualquier persona en el momento de aplicar de un modo flexible fórmulas de saber y de saber hacer debidamente contextualizadas (Poblete y García, 2007), la segunda dejaría de manifiesto y reconocería de una manera sistematizada la experiencia con la que ingresan los estudiantes en la universidad y que, de hecho, siguen adquiriendo mientras desarrollan los estudios universitarios.

\subsection{Perspectivas de enseñanza y e-portafolios}

Las perspectivas de portafolios que antes enunciamos orientan en relación también a las metodologías que asumimos desde la enseñanza. El portafolios se transforma en un recurso que posibilita que el alumnado muestre y transforme en evidencias aquello que ha aprendido; por lo tanto, la propuesta de enseñanza tiene que ofrecer alternativas a la búsqueda autónoma de significados construidos por el sujeto, a la propia estructuración conceptual de los contenidos y al enriquecimiento con la integración de experiencias que devienen de diversos contextos. De esta forma, cada portafolios es único y muestra al sujeto que lo asume tal como es. Este margen de libertad, de expresión y auto-expresión puede ser idiosincrásico de los enfoques de portafolios que estamos presentando, también para que sean reflejo de una evaluación auténtica.

En ese marco, el profesor toma decisiones sobre la enseñanza que realiza, y planifica el tipo de portafolios que le solicita al alumno, el lugar que ocupará en el proceso su elaboración y producción y también la manera en que se abordará su evaluación. En la medida que forma parte de la propuesta de enseñanza, el portafolio comienza a ocupar un espacio en cada uno de sus momentos (planificación, desarrollo, evaluación). Es allí donde juegan un rol significativo las reflexiones en torno a: por qué y para qué el portafolios, sus características y formato, los elementos a incorporar, criterios de evaluación, planificación de los tiempos (alumnos y profesores) y organización del análisis y feedback (cuándo y cómo).

Con esto estamos queriendo apuntar que introducir una herramienta como el portafolios implica de alguna manera volver a reflexionar sobre la enseñanza que se practica, globalmente entendida: desde la selección apropiada de contenidos, hasta las propuestas de técnicas, procedimientos y recursos más adecuados y/o coherentes con ella.

Ahora bien, es posible que el profesorado adapte la herramienta a lo que ya hace, incorporando algunos aspectos de la misma en función de las teorías que regulan su enseñanza, sin replantearse el conjunto. En este sentido, el portafolios puede mostrarse como un reflejo de la perspectiva de enseñanza que adopta el profesor y de allí que observando qué portafolios solicita al alumnado, podríamos descubrir cuál es la idea de enseñanza que tiene este profesor y también qué concepción de conocimiento está sustentando en la propuesta.

Parafraseando las famosas metáforas que Wilson (1995) ilustró hace tiempo, podemos decir que

Portafolios electrónicos y educación superior en España: Situación y tendencias. 


\begin{tabular}{|l|l|}
\hline Si piensas la enseñanza como... & Entonces piensas el portafolios como ... \\
\hline Un producto para ser transportado & $\begin{array}{l}\text { Un conjunto de ejercicios y/o elementos pre- } \\
\text { definidos por el profesor que reproducen el } \\
\text { producto que se ha trasmitido. }\end{array}$ \\
\hline $\begin{array}{l}\text { Un conjunto de estrategias que pretenden } \\
\text { cambiar y ampliar los esquemas de los } \\
\text { individuos }\end{array}$ & $\begin{array}{l}\text { Un sistema de evidencias seleccionas por el } \\
\text { alumno/a como resultado de la reflexión sobre } \\
\text { su propio proceso de aprendizaje }\end{array}$ \\
\hline $\begin{array}{l}\text { Un conjunto de estrategias que requiere la } \\
\text { participación en la comunidad en las actividades } \\
\text { cotidianas }\end{array}$ & $\begin{array}{l}\text { Un sistema de evidencias seleccionadas por el } \\
\text { alumno/a reflexionadas y compartidas en } \\
\text { comunidad, compartiendo opiniones, ideas y } \\
\text { recursos. }\end{array}$ \\
\hline
\end{tabular}

Como toda clasificación, la anterior tiene el pecado original de mostrarse rígida y forzada. La realidad es mucho más neutral y puede ofrecernos versiones híbridas de las diferentes variantes. Si bien podemos decir que un conjunto de ejercicios solicitados por el profesor a todo el alumnado, respondería a la versión de la primera fila de esa tabla y -en alguna medida podríamos pensar que tergiversa las representaciones desde donde se ha configurado la herramienta portafolios-, sí podríamos afirmar que el conjunto de esos ejercicios, con la reflexión del alumno en relación al aprendizaje que le ha significado su realización y también, el proceso que le ha llevado a su resolución, incluyendo los errores como fuente de aprendizaje, podrían ser elementos que se incluyan en una propuesta como la que se representa en la segunda y tercera fila de la tabla anterior.

\section{LINEAS DE FUTURO}

Después de este recorrido por las diferentes perspectivas con las que nos podemos encontrar al analizar el uso de e-portafolios en el sistema universitario, se pone de manifiesto la necesidad de abordar las líneas de futuro. Tanto los desarrollos posteriores que puede tener el sistema como las direcciones que pueden tomar los mismos.

Siempre es arriesgado indicar el camino que va a seguir una tecnología/pedagogía como son los e-portafolios. Hace unos años no existían las herramientas denominadas ahora de Web 2.0 y, de manera segura, dentro de otros pocos años habrán aparecido otras completamente nuevas que modificarán las prácticas $\mathrm{y}$, también, nuestra percepción del sentido y finalidades de los portafolios digitales. Para realizar las tareas básicas de seleccionar y agrupar evidencias, mostrarlas, y poder dialogar sobre ellas (y otras acciones más, desde luego), muchas tecnologías son posibles, al igual que existen muchas maneras de organizar el e-portafolio educativamente. Dada su distinta naturaleza cada una aporta funcionalidades y limitaciones de manera simultánea.

Nos parece que hay, como mínimo, tres grandes ámbitos que marcarán lo que va a ocurrir en los próximos años, tanto en el contexto universitario como en el más general de la sociedad: A) el desarrollo tecnológico de los portafolios, tal y como son ahora pensados; B) los cambios educativos e institucionales y, C) los cambios sociales en el 
uso de Internet y de las tecnologías de la información. Los revisaremos únicamente en algún aspecto que nos parece especialmente destacado.

A) Cuando se revisa el estado actual del uso de los portafolios (Jafari y Kaufman, 2006), surge siempre la cuestión de su diversidad. Tanto en las plataformas que se utilizan como en las prácticas asociadas, sea en la universidad, o en otros contextos educativos o más generales. No es posible en estos momentos, ni quizá deseable, que se produzca un proceso de estandarización de las soluciones tecnológicas ni, desde luego, de las prácticas educativas. Sin embargo, parece del todo deseable que los diferentes sistemas de portafolios puedan comunicarse entre sí (lo que no ocurre tampoco en estos momentos), sobre todo en beneficio de los propios usuarios, sean estudiantes, profesores, o ciudadanos.

Esta problemática se conoce con un nombre técnico, la interoperabilidad, y todavía está lejos de alcanzarse (IMS, 2005). Que los sistemas sean interoperables quiere decir, en esencia, que sean capaces de intercambiar datos de manera estructurada: que un portafolios de un estudiante de la Universidad 1, realizado con el sistema de portafolios alfa, pueda ser exportado y reimportado en la Universidad 2 que tiene un sistema de portafolios beta. O que cualquiera de ambos sistemas pueda enviar sus datos a otro sistema situado en otro contexto de uso de portafolios, por ejemplo no universitario como la transición al mundo laboral. Se comprende el interés de esta situación para las personas que han construido sus portafolios, a lo largo de los años, y que quieren conservarlos en otras situaciones y para otros propósitos.

La interoperabilidad, para la que todavía no hay definidos estándares de intercambio de manera consensuada, conlleva un ideal que los portafolios "en papel" siempre han tenido, pues las carpetas permanecían a través de las situaciones aunque hubiera que reorganizarlas. Por otra parte, puede tener un lado más complejo asociado al riesgo de que datos personales se muevan entre distintos sistemas, por lo que parece necesario asociarla con problemáticas de privacidad y de seguridad.

B) Los cambios educativos e institucionales se refieren al núcleo de los usos universitarios. En general, las universidades están optando por utilizar sistemas/plataformas de aprendizaje virtual (o su acrónimo en inglés $V L E$ ) como medios para modalidades de enseñanza a distancia e híbrida. Estos sistemas ofrecen muchas ventajas, sobre todo si los consideramos desde el punto de vista de su gestión, entre ellas la de tener una administración centralizada de todo el sistema, así como la de ser herramientas pensadas desde el punto de vista del profesor que tiene su "aula virtual". Es decir, su unidad de trabajo es el curso o asignatura.

Los portafolios se mueven en ese terreno como una realidad en ascenso (es suficiente con ver las aportaciones a este monográfico) y también como una gran promesa, sobre todo en aspectos de evaluación, continuada y auténtica, así como de aprovechar las evidencias de aprendizaje para generar nuevos aprendizajes mediante la reflexión y el diálogo — aunque todavía sin acabar de incluir funciones de acreditación de manera habitual.

Pero han surgido alternativas a los portafolios y a los VLE, mayoritariamente en ámbitos personales y no institucionales, que probablemente irán aumentando en el futuro. Una de ellas son los denominados "Entornos Personales de Aprendizaje" (o $P L E$ ), fruto de las nuevas aplicaciones basadas en Internet, que permiten conectar un buen número de entornos de información y de comunicación, que las personas utilizan, cada vez más, en su vida cotidiana. Incluso han aparecido "integradores" de estos 
entornos, como el navegador Flock que permite visualizar muchas de esas herramientas o entornos en una única ventana.

C) Los PLE son un reflejo de cómo está cambiando el uso de Internet, la denominada "web social" y las múltiples aplicaciones que se utilizan. El papel de los portafolios en ese nuevo espacio seguramente se tiene que redefinir, pues muchas de estas aplicaciones son utilizadas para conservar archivos y materiales que sus usuarios consideran importantes, y que son una muestra de sus intereses, e incluso de los logros alcanzados previamente, así como de las redes sociales virtuales en las que participan. De hecho, existe una tensión entre los usos institucionales de los VLE, e incluso de los portafolios académicos, y el conjunto de aplicaciones personales y sociales que se utilizan sobre todo fuera de los ambientes universitarios, y que ha crecido de manera exponencial en estos últimos años.

En resumen, junto a una necesidad de comunicación entre los sistemas de eportfolios, y de los intereses institucionales que la amparan, los cambios sociales, personales y tecnológicos parecen dibujar un panorama altamente abierto y con muchas alternativas funcionales. No es difícil pensar que los portafolios, académicos y personales, ocuparán un lugar muy importante en ese entramado, sobre todo si actúan como puentes o conectores entre las necesidades de las instituciones académicas, preocupadas por cuestiones de acreditación y de evaluación, las de los estudiantes que mantendrán sus propios espacios de información, comunicación y aprendizaje, y las de los profesores que buscan herramientas y metodologías sostenibles en un entorno de enseñanza, virtual y presencial, cada vez más complejo.

Presentación del artículo original: 1 de abril de 2009

Fecha de aprobación: 15 de abril de 2009

Fecha de publicación: 15 de julio de 2016

Barberà, E. et al (2016). Portafolios electrónicos y educación superior en España: Situación y tendencias. RED. Revista de Educación a Distancia, 50. Consultado el (dd/mm/aaaa) en http://www.um.es/ead/red/50

\section{Referencias}

Barberà, E. (2008). El estilo e-portafolio. Barcelona. Editorial UOC.

Barrett, H. (1998). Strategic questions to consider when planning for electronic portfolios. Leaning \& Leading with technology, 26(2), 6-13

Barrett, H. and Wilkerson, J. (2004). Conflicting Paradigms in Electronic Portfolio Approaches. Consultado 11/03/2008 en:

http://electronicportfolios.org/systems/paradigms.html

Blackburn, J. L.; Hakel, M. D. (2006). Enhancing self-regulation and goal orientation with eportfolios. In A. Jafari; C. Kaufman (Eds.), Handbook of Research on ePortfolios (83-89). Hershey PA: Idea Group Reference. 
IMS (2005). IMS ePortfolio Best Practice and Implementation Guide. En línea: http://www.imsglobal.org/ep/epv1p0/imsep_bestv1p0.html.[Consultado: 14/07/2008]

Jafari, A. \& Kaufman, C. (eds, 2006). Handbook of Research on ePortfolios. Hershey (PA): Idea Group.

Litwin, E. (1997). Las configuraciones didácticas. Una nueva agenda para la enseñanza superior. Buenos Aires: Paidós.

Morris, J, \& Buckland, H. (2000). Electronic Portfolios for Learning and Assessment. Proceedings: Society for Information Technology and Teacher Education (SITE) San Diego CA, pp. 1085-1090.

Poblete, M. y García Olalla, A. (Coord.) (2007). Desarrollo de competencias y créditos transferibles: experiencia multidisciplinar en el contexto universitario. Madrid: Mensajero.

Shulman, L. (1999). Portafolios del docente: una actividad teórica. En N. L. (Comp.) (Ed.), El uso del portafolios. Propuestas par un nuevo profesionalismo docente. (45-62). Buenos Aires: Amorrortu.

Wiedmer, T. L. (1998). Digital portfolios. Phi Delta Kappan, 70 (8), 586-590.

Wilson, B. (1995). Metaphors for instruction: Why we talk about learning environments. Educational Technology, Special Seccion: Constructivist learning environments, september-october, 25-30. 